J. Lake Sci. (湖泊科学), 2016, 28(3): 494-501

DOI 10. 18307/2016. 0304

(C) 2016 by Journal of Lake Sciences

\title{
江苏省五大湖泊水体重金属的监测与比较分析
}

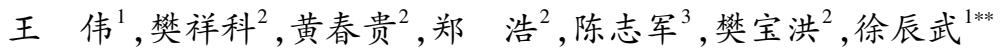 \\ (1:扬州大学农学院,扬州 225009) \\ (2: 江苏省渔业生态环境监测站,南京 210036) \\ (3: 中国农业科学院农业质量标准与检测技术研究所,北京 100081)
}

\begin{abstract}
摘 要: 2001-2011 年(不含 2004 年) 10 年间对江苏省五大湖泊 (太湖、滆湖、洪泽湖、高宝邵伯湖和骆马湖) 的水体重金 属浓度进行长期定点监测, 按系统分组资料进行方差分析, 以比较各重金属浓度在湖泊间的差异显著性,并在此基础上 应用多指标综合评价法 (TOPSIS 法) 对 5 个湖泊水体重金属进行综合比较. 结果表明: 除铜浓度外, 5 个湖泊间铅、镉、录 和砷 4 个指标浓度均存在极显著差异; 5 种重金属浓度湖泊内站位间均差异不显著; 铅、镉和录浓度站位内年份间差异显 著或极显著. TOPSIS 分析结果表明, 总体而言高宝邵伯湖水体重金属污染程度最小, 骆马湖水体重金属污染程度最大,洪 泽湖、太湖和滆湖水体重金属污染综合评价优劣相当.
\end{abstract}

关键词: 渔业水质;重金属;太湖;滆湖;洪泽湖;高宝邵伯湖;骆马湖

\section{Monitoring and comparison analysis of heavy metals in the five great lakes in Jiangsu Province}

WANG Wei ${ }^{1}$, FAN Xiangke ${ }^{2}$, HUANG Chungui ${ }^{2}$, ZHENG $\mathrm{Hao}^{2}$, CHEN Zhijun ${ }^{3}$, FAN Baohong ${ }^{2} \&$ XU Chen$\mathrm{wu}^{1 * *}$

(1: College of Agriculture, Yangzhou University, Yangzhou 225009, P.R. China)

(2: Jiangsu Fishery Environment Monitoring Center, Nanjing 210036, P.R. China)

(3: Institute of Quality Standards and Testing Technology for Agro-Products, Chinese Academy of Agricultural Sciences, Beijing 100081, P.R. China)

Abstract: The long-term localization monitors on heavy metals during the past 10 years from 2001 to 2011 ( except 2004 ) were carried out in water of the five great lakes, including Lakes Taihu, Gehu, Hongze, Gaobaoshaobo and Luoma in Jiangsu Province. Nested data variance analysis was performed in order to compare the difference of heavy metals among the five above-mentioned lakes, and the Technique for Order Preference by Similarity to Ideal Solution( TOPSIS ) method was employed to give a comprehensive assessment of heavy metals. The results indicated that concentrations of $\mathrm{Pb}, \mathrm{Cd}, \mathrm{Hg}$ and As were all very significantly different compared within the lakes except $\mathrm{Cu}$. In addition, the concentrations of the five heavy metals at each monitor station were not significant. Concentrations of $\mathrm{Pb}, \mathrm{Cd}$ and $\mathrm{Hg}$ were significant or very significantly different among years for each station. The TOPSIS results showed that the pollution of heavy metals in water of Lake Gaobaoshaobo was the smallest but was the greatest in Lake Luoma. For the remaining three lakes, the pollutions of heavy metals were almost at the same level.

Keywords: Fishery water; heavy metal; Lake Taihu; Lake Gehu; Lake Hongze; Lake Gaobaoshaobo; Lake Luoma

湖泊是地表生态系统中人类赖以生存的自然单元之一,在供水、防洪、养殖、旅游、航运和维持生态平衡 等方面发挥着巨大的作用. 数十年来, 随着我国人口增加和工农业生产的发展, 加之湖泊水资源的过度开

＊江苏省高校 “青蓝工程” 科技创新团队、江苏省作物学优势学科、农业部农业财政专项项目和江苏省农业科技自主 创新资金项目 (CX (14) 2094) 联合资助. 2014-09-19 收稿;2015-09-15 收修改稿. 王伟(1979 ), 女,博士研究 生; E-mail: stddev@163.com.

** 通信作者; E-mail: cwxu@yzu.edu.cn. 
发,使得湖泊水资源短缺、水环境恶化和生态系统退化等问题日益凸显,已严重威胁到社会经济的可持续发 展和人类健康 ${ }^{[1]}$.

水环境是一个开放和动态的体系, 其中生物与非生物环境是相互关联和相互作用的 ${ }^{[2]}$. 随着现代物质 文明的飞速发展, 人类活动向水环境中排放的污染物中包含的重金属也日益增多. 重金属元素在水环境中 的累积, 对水生动物的生长发育、种族繁衍等一系列生命活动造成了极大的威胁. 如重金属永 $(\mathrm{Hg}) 、$ 镉 $(\mathrm{Cd}) 、$ 铜 $(\mathrm{Cu})$ 等, 其污染特点是来源广、残留时间长、具有累积性、能沿食物链转移、污染后不易被发现和难 以恢复等,严重地危害着渔业水环境和水生生物,也对人类健康造成潜在威胁.

江苏是我国淡水湖泊分布集中的省 (区) 之一. 现有太湖、滆湖、洪泽湖、高宝邵伯湖和骆马湖 5 个省管 湖泊, 其中对于太湖和洪泽湖的研究是国内研究的热点 ${ }^{[3-9]}$. 多年来, 主管部门虽然对这五大湖泊的增殖区、 保护区、网围养殖区和进出湖河道等功能区开展渔业环境监测, 但对这 5 个湖泊大量的渔业水质监测数据 还缺乏系统分析. 因此, 采用现代统计分析技术从这些监测数据中挖掘出该地区湖泊水质的变化规律, 不但 可以为当地渔业生产服务,还可以为该地区行业发展政策制定提供技术支撑.

多指标综合评价法 (Technique for Order Preference by Similarity to Ideal Solution,TOPSIS) 也叫逼近于理 想解的技术, 由 Hwang 和 Yoon 于 1981 年首次提出, 是系统工程中有限方案多目标决策分析常用的决策方 法, 其方法的核心思想是通过先定义决策问题的理想解与负理想解, 然后比较评价方案与理想解和负理想 解的距离远近, 最后计算各个方案与理想解的相对贴近度, 进行方案的优劣排序. TOPSIS 法对数据分布及 样本量、指标多少无严格限制, 数学计算亦不复杂, 既适用于少样本资料, 又适用于多样本的大系统, 已在医 疗、经济、农业等领域中得到广泛应用 ${ }^{[10-11]}$. 以往的渔业水质监测数据仅以简单的柱形图描述各监测指标是 否超标, 无统计学证据支撑. 目前, 基于长期定位监测数据的江苏省主要湖泊水质重金属的比较分析与综合 评价,亦未见相关文献报道.

本文首次利用系统分组资料方差分析方法对江苏多年定位监测的 5 个湖泊水体的重金属数据进行统 计分析, 以鉴别湖泊间、湖泊内站位间和站位内年份间的差异显著性, 在此基础上应用 TOPSIS 法对 5 个湖 泊水体重金属进行综合对比分析,并对它们的变化规律进行总结.

\section{1 材料与方法}

\section{1 数据来源}

2001-2011 年(不含 2004 年) 间, 由江苏省渔业生态环境监测站统一采集水样. 采样时间上半年在 4 月 份,下半年在 10 月份, 5 个湖泊采样时间基本一致. 取重金属水样 $500 \mathrm{ml}$ 放于洁净的聚乙烯瓶中,之后加人 适量硝酸保存, 同时用采集的空白样品和 $10 \%$ 以上的平行样进行质量控制 ( QC 控制). 其中, 太湖共监测 413 站次, 滆湖共监测 183 站次, 洪泽湖共监测 205 站次, 高宝邵伯湖共监测 221 站次, 骆马湖共监测 142 站 次. 其监测站位见图 1.

\section{2 样品测定}

样品在硫酸一过硫酸钾体系中消解后, 测定 $\mathrm{Hg}$ 浓度, 加人抗坏血酸一硫腿后测定 As 浓度, 两者均采用 AFS-230a 原子苂光光度计测定. 采用 SpectrAA220 原子吸收分光光度计测定 $\mathrm{Cu} 、 \mathrm{~Pb}$ 和 $\mathrm{Cd}$ 浓度. 分析质量使 用试剂空白及平行样进行控制,加标回收来控制样品测定的准确性,结果均符合质控要求.

\section{3 统计分析方法}

1.3.1 系统分组资料方差分析 以湖泊作为组, 湖泊内监测站位作为组内亚组, 站位内年份作为小亚组, 进 行三级系统分组资料方差分析以比较各重金属指标在不同湖泊间的差异显著性.

1.3. 2 TOPSIS 法进行水质综合评价 主要分析步骤: (1) 建立同趋势化的原始数据矩阵, 以消除不同指标不 同量纲及其数量级的差异对评价结果的影响; (2) 对同趋势化的原始数据矩阵进行归一化处理, 并建立归一 化数据矩阵, 其计算公式为: $u=\left(x-x_{\text {min }}\right) /\left(x_{\text {max }}-x_{\text {min }}\right) ;(3)$ 根据归一化矩阵得到最优值向量 $\mathrm{A}^{+}$和最劣值 向量 $\mathrm{A}^{-} ;(4)$ 分别计算各方案的评价指标值与最优方案和最劣方案的距离 $D_{i}^{+}$和 $D_{i}^{-}$, 计算公式分别为: $D_{i}^{+}=$ $\sqrt{\sum\left(a_{i j \max }-a_{i j}\right)^{2}}$ 和 $D_{i}^{-}=\sqrt{\sum\left(a_{i j \mathrm{~min}}-a_{i j}\right)^{2}}$; (5) 计算各评价指标与最优方案的接近程度, 计算公式为: $C_{i}=$ $D^{-} /\left(D^{-}+D^{+}\right)$. 

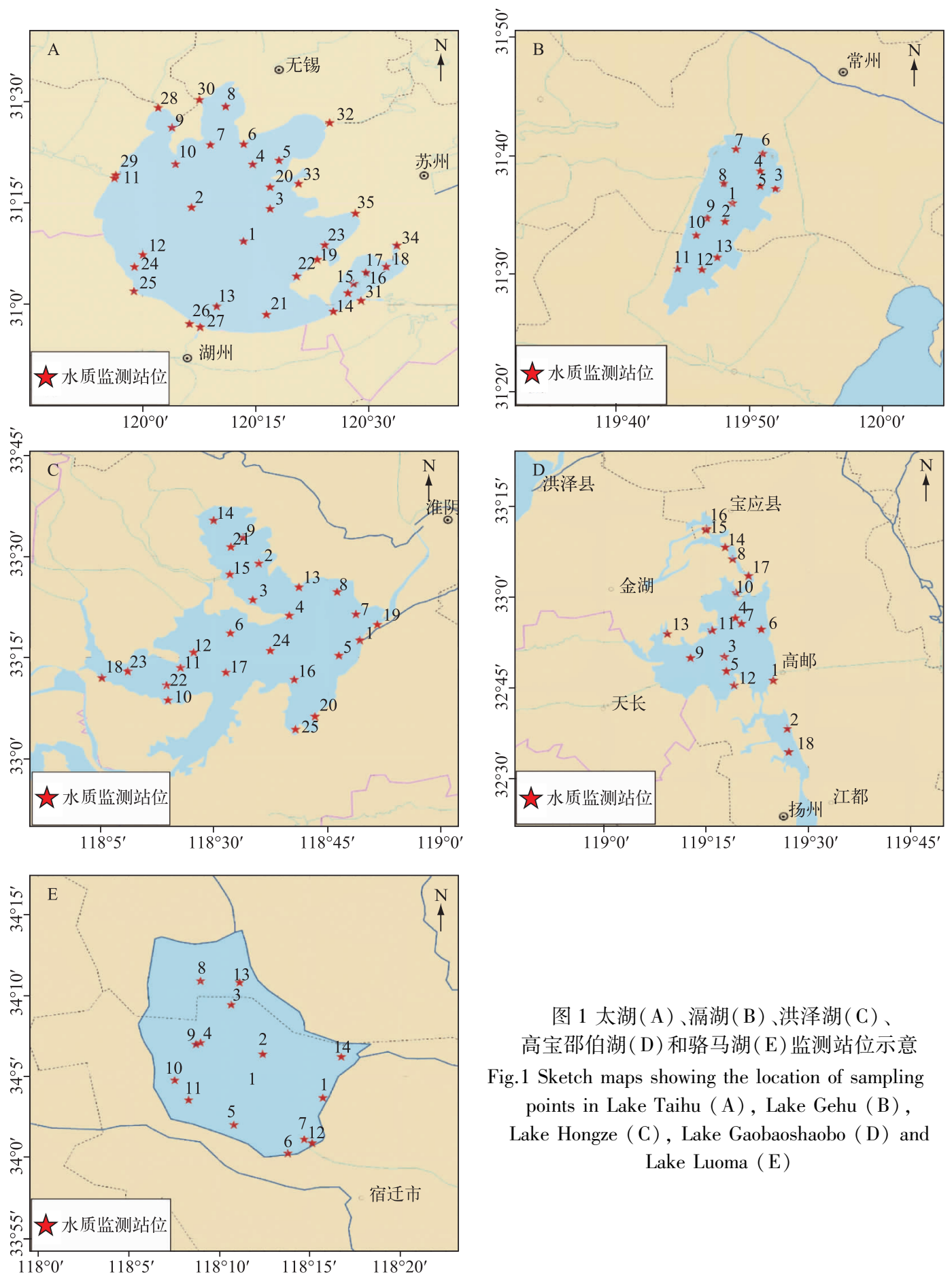

图 1 太湖 $(A)$ 、滆湖 $(B)$ 、洪泽湖 $(\mathrm{C})$ 、 高宝邵伯湖 $(D)$ 和骆马湖 $(E)$ 监测站位示意 Fig.1 Sketch maps showing the location of sampling points in Lake Taihu (A), Lake Gehu (B), Lake Hongze ( C), Lake Gaobaoshaobo (D) and Lake Luoma (E)

\section{2 结果与分析}

\subsection{0 年内五大湖泊重金属分布特征}

10 年内, 江苏省五大湖泊 $\mathrm{Cu}$ 浓度的平均数为 $4.54 \mu \mathrm{g} / \mathrm{L}$, 中位数为 $2.86 \mu \mathrm{g} / \mathrm{L}$, 众数为 $2.00 \mu \mathrm{g} / \mathrm{L} ; \mathrm{Pb}$ 
浓度平均数为 $4.03 \mu \mathrm{g} / \mathrm{L}$, 中位数为 $2.50 \mu \mathrm{g} / \mathrm{L}$, 众数为 $0.50 \mu \mathrm{g} / \mathrm{L}$; Cd 浓度平均数为 $1.02 \mu \mathrm{g} / \mathrm{L}$, 中位数为 $0.50 \mu \mathrm{g} / \mathrm{L}$, 众数为 $0.005 \mu \mathrm{g} / \mathrm{L} ; \mathrm{Hg}$ 浓度平均数为 $0.118 \mu \mathrm{g} / \mathrm{L}$, 中位数为 $0.075 \mu \mathrm{g} / \mathrm{L}$, 众数为 $0.062 \mu \mathrm{g} / \mathrm{L}$; 砷 浓度平均数为 $2.84 \mu \mathrm{g} / \mathrm{L}$, 中位数为 $2.66 \mu \mathrm{g} / \mathrm{L}$, 众数为 $2.03 \mu \mathrm{g} / \mathrm{L}$. 汇总五大湖泊水体重金属的数据, 其中均 是众数 $<$ 中位数 $<$ 平均数, 可知 5 种重金属浓度呈右偏态分布 $($ 图 2).
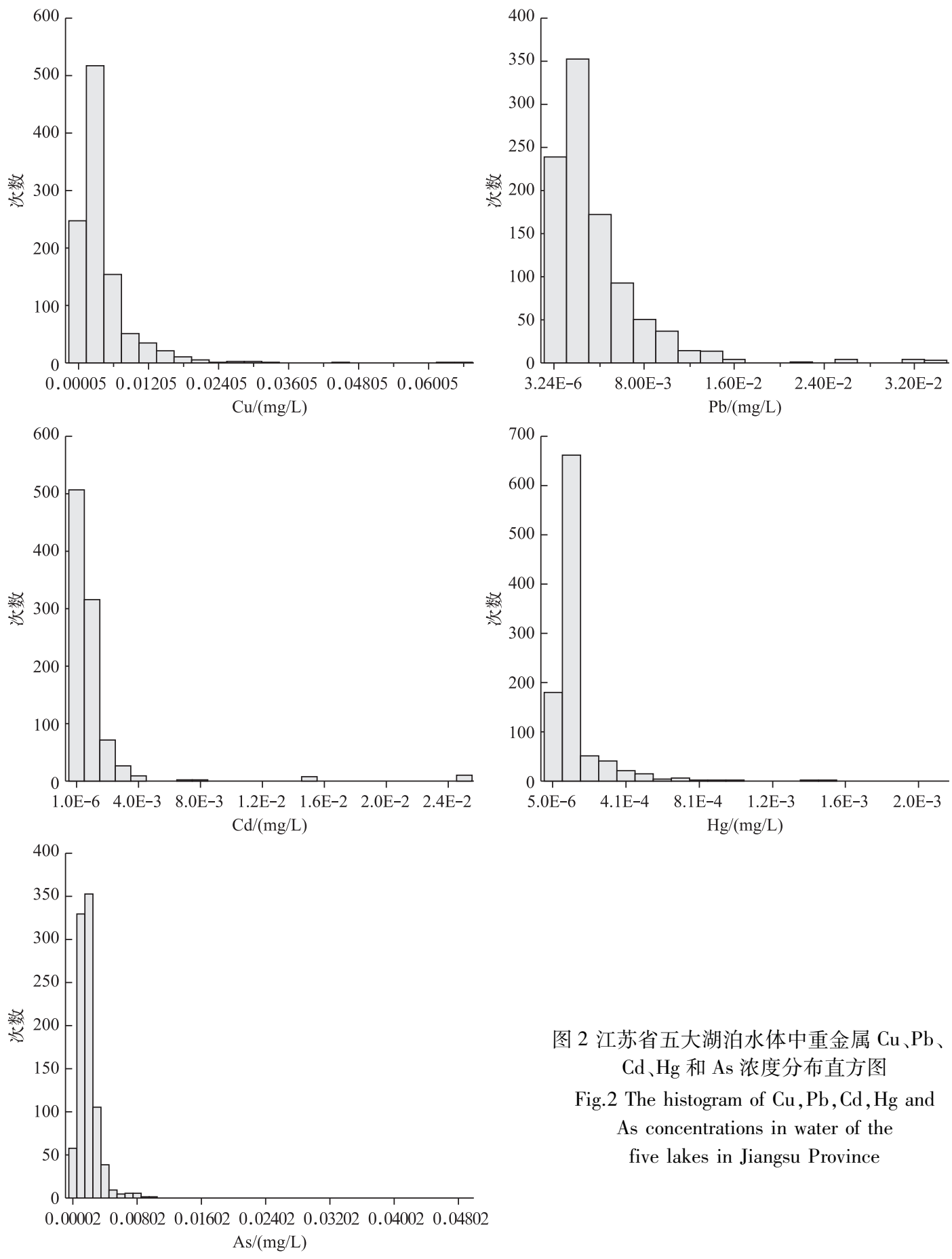

图 2 江苏省五大湖泊水体中重金属 $\mathrm{Cu} 、 \mathrm{~Pb}$ 、 $\mathrm{Cd} 、 \mathrm{Hg}$ 和 $\mathrm{As}$ 浓度分布直方图

Fig. 2 The histogram of $\mathrm{Cu}, \mathrm{Pb}, \mathrm{Cd}, \mathrm{Hg}$ and As concentrations in water of the five lakes in Jiangsu Province 


\section{2 五大湖泊渔业水质重金属指标的理化特征}

分别对不同湖泊水体重金属监测数据进行描述性统计分析,可以得到其指标特征 (表 1).

由表 1 可知,江苏省五大湖泊 10 年间水体重金属 $\mathrm{Cu} 、 \mathrm{~Pb} 、 \mathrm{Cd} 、 \mathrm{Hg} 、 \mathrm{As}$ 的平均浓度均符合《渔业水质标 准》 ${ }^{[12]}$, 但是相对变异均较大, 尤其是 $\mathrm{Cu}$ 浓度的最大值已超过《渔业水质标准》 ${ }^{[12]}$. 说明重金属监测过程中 个别年份个别站位重金属污染较重, 存在极端大值的影响.

表 $12001-2011$ 年江苏省五大湖泊水体中重金属浓度基本统计量

Tab.1 Basic statistics of heavy metal concentrations in water of the five lakes in Jiangsu Province from 2001 to 2011

\begin{tabular}{|c|c|c|c|c|c|c|}
\hline 湖泊 & 统计量 & $\mathrm{Cu} /(\mu \mathrm{g} / \mathrm{L})$ & $\mathrm{Pb} /(\mu \mathrm{g} / \mathrm{L})$ & $\mathrm{Cd} /(\mu \mathrm{g} / \mathrm{L})$ & $\mathrm{Hg} /(\mu \mathrm{g} / \mathrm{L})$ & $\mathrm{As} /(\mu \mathrm{g} / \mathrm{L})$ \\
\hline \multirow[t]{5}{*}{ 太湖 } & 最小值 & 1.46 & 1.03 & 0.06 & 0.03 & 0.48 \\
\hline & 最大值 & 28. 00 & 6.31 & 1.46 & 0.14 & 13. 25 \\
\hline & 平均值 & 5.72 & 3.84 & 1.03 & 0.08 & 10.40 \\
\hline & 标准差 & 5.49 & 3. 15 & 0.75 & 0.07 & 9.67 \\
\hline & 变异系数/\% & 95.98 & 82.03 & 72.82 & 87.50 & 92.98 \\
\hline \multirow[t]{5}{*}{ 滆湖 } & 最小值 & 1.92 & 1.53 & 0.04 & 0.02 & 0.60 \\
\hline & 最大值 & 12. 80 & 6.71 & 1.53 & 0.19 & 4. 08 \\
\hline & 平均值 & 5.89 & 3.46 & 0.75 & 0.15 & 2. 61 \\
\hline & 标准差 & 5. 12 & 3.01 & 0.67 & 0.14 & 1.75 \\
\hline & 变异系数/\% & 86.93 & 86.99 & 89.33 & 93.33 & 67.05 \\
\hline \multirow[t]{5}{*}{ 洪泽湖 } & 最小值 & 1.00 & 1.00 & 0.05 & 0.04 & 0.41 \\
\hline & 最大值 & 22.75 & 5.11 & 2. 03 & 0.25 & 7. 02 \\
\hline & 平均值 & 4. 99 & 3.70 & 1.44 & 0.17 & 1.77 \\
\hline & 标准差 & 4. 84 & 3. 40 & 1.38 & 0.15 & 1.04 \\
\hline & 变异系数/\% & 96.99 & 91.89 & 95.83 & 88.24 & 58.76 \\
\hline \multirow[t]{5}{*}{ 高宝邵伯湖 } & 最小值 & 1.25 & 1.50 & 0.10 & 0.03 & 0.41 \\
\hline & 最大值 & 17.67 & 5.50 & 1. 49 & 0.16 & 8.31 \\
\hline & 平均值 & 4. 26 & 3.27 & 1. 19 & 0.07 & 1.68 \\
\hline & 标准差 & 3.79 & 3.04 & 1.09 & 0.02 & 0.76 \\
\hline & 变异系数/\% & 88.97 & 92.97 & 91.60 & 28.57 & 45.24 \\
\hline \multirow[t]{5}{*}{ 骆马湖 } & 最小值 & 1.50 & 0.50 & 0.04 & 0.03 & 0.31 \\
\hline & 最大值 & 12.00 & 15. 18 & 1.67 & 0.24 & 3.41 \\
\hline & 平均值 & 3.73 & 8.54 & 0.23 & 0.19 & 1.15 \\
\hline & 标准差 & 3.21 & 8.20 & 0.20 & 0.16 & 0.66 \\
\hline & 变异系数/\% & 86.06 & 96.02 & 86.96 & 84.21 & 57.39 \\
\hline 《渔业水质标准》 ${ }^{[12]}$ & & 10 & 50 & 5 & 0.5 & 50 \\
\hline
\end{tabular}

\section{3 五大湖泊水体重金属指标差异显著性比较}

采用系统分组资料方差分析方法对上述重金属指标进行方差分析,结果表明: 湖泊水体中除 $\mathrm{Cu}$ 浓度 外,其他 4 种重金属浓度 5 个湖泊间均差异极显著,这可能与湖泊周围环境条件的差异、入湖污染物的排放 以及农田农药化肥的施用有关. 另外, 湖泊内站位间各重金属浓度的差异均不显著. $\mathrm{Pb} 、 \mathrm{Cd} 、 \mathrm{Hg}$ 浓度站位内 年份间差异显著或极显著,说明它们年际变化均不稳定, $\mathrm{Cu}$ 和 $\mathrm{As}$ 浓度站位内年份间差异不显著(表 2).

进一步对湖泊间差异显著或极显著的重金属浓度进行多重比较,结果见表 3.

多重比较结果表明: 骆马湖水体 $\mathrm{Pb}$ 浓度最高, 为 $8.54 \mu \mathrm{g} / \mathrm{L}$, 与其他 4 个湖泊间差异显著. 洪泽湖 $\mathrm{Cd}$ 浓 度最高为 $1.44 \mu \mathrm{g} / \mathrm{L}$, 与太湖和高宝邵伯湖差异不显著, 与滆湖和骆马湖差异显著. 骆马湖 $\mathrm{Hg}$ 浓度最高为 $0.19 \mu \mathrm{g} / \mathrm{L}$, 与洪泽湖差异不显著, 与其他 3 个湖泊差异显著. 太湖 As 浓度最高为 $10.40 \mu \mathrm{g} / \mathrm{L}$, 与其他 4 个 湖泊差异显著,其他 4 个湖泊间差异不显著. 
表 2 江苏省五大湖泊水体中重金属浓度方差分析结果 ${ }^{1)}$

Tab.2 The ANOVA of heavy metal concentrations in water of the five lakes in Jiangsu Province

\begin{tabular}{|c|c|c|c|c|c|c|c|c|c|c|c|c|c|c|c|}
\hline \multirow{2}{*}{ 变异来源 } & \multicolumn{3}{|c|}{$\mathrm{Cu}$} & \multicolumn{3}{|c|}{$\mathrm{Pb}$} & \multicolumn{3}{|c|}{$\mathrm{Cd}$} & \multicolumn{3}{|c|}{$\mathrm{Hg}$} & \multicolumn{3}{|c|}{ As } \\
\hline & $\mathrm{d} f$ & $M S$ & $F$ & $\mathrm{~d} f$ & MS & $F$ & $d f$ & MS & $F$ & $\mathrm{~d} f$ & MS & $F$ & $\mathrm{~d} f$ & MS & $F$ \\
\hline 湖泊间 & 4 & 1. 56E-4 & 0.66 & 4 & 6. $23 \mathrm{E}-4$ & 33. $97^{\text {** }}$ & 4 & 3. $29 \mathrm{E}-5$ & $8.92^{* *}$ & 4 & 4. $89 \mathrm{E}-71$ & $19.92^{\text {** }}$ & 4 & 3. $70 \mathrm{E}-3$ & $5.25^{* *}$ \\
\hline 湖泊内站位间 & 月 113 & 2. $38 \mathrm{E}-4$ & 0.96 & 110 & 1. $84 \mathrm{E}-5$ & 0.32 & 108 & 3. $69 \mathrm{E}-6$ & 0.41 & 114 & 2. $46 \mathrm{E}-8$ & 0.77 & 113 & 7. $04 \mathrm{E}-4$ & 0.56 \\
\hline 站位内年份间 & 月 351 & 2. $47 \mathrm{E}-4$ & 1.08 & 340 & 5. 79E-5 & $5.68^{* *}$ & 328 & 8. $94 \mathrm{E}-6$ & $1.19^{*}$ & 328 & 3. $00 \mathrm{E}-8$ & $1.50^{\text {** }}$ & 328 & 1. $26 \mathrm{E}-3$ & 1.14 \\
\hline 误差 & 596 & 2. $29 \mathrm{E}-4$ & & 550 & 1. $02 \mathrm{E}-5$ & & 503 & 7. $48 \mathrm{E}-6$ & & 539 & 2. $00 \mathrm{E}-8$ & & 471 & 1. $10 \mathrm{E}-3$ & \\
\hline 总变异 & 1064 & & & 1004 & & & 943 & & & 985 & & & 916 & & \\
\hline
\end{tabular}

1) $\mathrm{d} f 、 M S 、 F$ 分别代表方差分析中的自由度、均方和测验统计量 $F$ 值; ** 表示差异极显著; * 表示差异显著.

\section{410 年内五大湖泊重金属变化趋势}

10 年间, $\mathrm{Cu}$ 浓度在江苏省五大湖泊间具有 相同的变化趋势, 5 个湖泊基本上在 2002 年前 后出现最大值, 近几年 $\mathrm{Cu}$ 浓度有所下降, 说明 可能 2002 年前后 5 个湖泊均有含 $\mathrm{Cu}$ 污染物的 排人, 导致 $\mathrm{Cu}$ 浓度的增高. As 浓度在 5 个湖泊 间也具有相同的变化趋势, 5 个湖泊基本上在 2006 年前后出现最大值, 近几年 As 浓度有所下 降, 说明在 2006 年前后 5 个湖泊可能均有含 $\mathrm{As}$ 污染物的排人, 随着水体的流动和更替, As 浓度 近几年来有所下降. 而 5 个湖泊 $\mathrm{Pb}$ 浓度 2007 年 以来相对较高, 其平均浓度明显高于 2006 年之 前. 5 个湖泊 2003-2006/2007 年 Hg 浓度也高 于其他年份. 10 年内 5 个湖泊 $\mathrm{Cd}$ 浓度变化趋势 无明显规律 (表 3).

\section{5 五大湖泊水质重金属综合评价}

采用 TOPSIS 法进行分析时,将同趋势化后 的数据进行归一化处理, 并建立相应的归一化 数据矩阵 (表 4$)$.

由表 4 可知最优值向量 $\mathrm{A}^{+}$和最劣值向量 $\mathrm{A}^{-}$分别为 $\mathrm{A}^{+}=\min (0,0,0,0,0)$ 和 $\mathrm{A}^{-}=\max (1$, $1,1,1,1)$.

计算各重金属指标值与最优方案和最劣方 案的距离, 以及与最优方案的相对接近程度 $\left(C_{i}\right)$,并按 $C_{i}$ 值大小对各水体进行排序. $C_{i}$ 值越 小, 说明重金属污染越轻; 反之, 污染越重. 由表 5 可知: 高宝邵伯湖 $C_{i}$ 值 $(0.5091)$ 最小, 骆马湖 $C_{i}$ 值 $(0.5858)$ 最大, 说明总体而言高宝邵伯湖 水体重金属污染程度最小, 骆马湖水体重金属 污染程度最大. 洪泽湖 $C_{i}$ 值 $(0.5226)$ 、太湖 $C_{i}$ 值 $(0.5397)$ 和滆湖 $C_{i}$ 值 $(0.5499)$ 相差不多, 说 明这 3 个湖泊重金属污染综合评价优劣相当.

表 3 江苏省五大湖泊渔业水质重金属浓度多重比较结果 Tab. 3 The multiple comparisons of heavy metal concentrations in fishery water of the five lakes in Jiangsu Province

\begin{tabular}{ccccr}
\hline 湖泊 & $\mathrm{Pb} /(\mu \mathrm{g} / \mathrm{L}) \mathrm{Cd} /(\mu \mathrm{g} / \mathrm{L}) \mathrm{Hg} /(\mu \mathrm{g} / \mathrm{L})$ & $\mathrm{As} /(\mu \mathrm{g} / \mathrm{L})$ \\
\hline 骆马湖 & $8.54^{\mathrm{a}}$ & $0.23^{\mathrm{c}}$ & $0.19^{\mathrm{a}}$ & $1.15^{\mathrm{b}}$ \\
太湖 & $3.84^{\mathrm{b}}$ & $1.03^{\mathrm{ab}}$ & $0.08^{\mathrm{c}}$ & $10.40^{\mathrm{a}}$ \\
洪泽湖 & $3.70^{\mathrm{b}}$ & $1.44^{\mathrm{a}}$ & $0.17^{\mathrm{ab}}$ & $1.77^{\mathrm{b}}$ \\
滆湖 & $3.46^{\mathrm{b}}$ & $0.75^{\mathrm{bc}}$ & $0.15^{\mathrm{b}}$ & $2.61^{\mathrm{b}}$ \\
高宝邵伯湖 & $3.27^{\mathrm{b}}$ & $1.19^{\mathrm{ab}}$ & $0.07^{\mathrm{c}}$ & $1.68^{\mathrm{b}}$ \\
\hline
\end{tabular}

$a 、 b 、 c$ 代表平均数间的差异显著性, 相同字母表示差异不显著, 不同字母表示差异显著.

表 4 江苏省五大湖泊水体中重金属指标归一化结果

Tab.4 The uniformization of heavy metal in water of the five lakes in Jiangsu Province

\begin{tabular}{ccccc}
\hline $\mathrm{Cu}$ & $\mathrm{Pb}$ & $\mathrm{Cd}$ & $\mathrm{Hg}$ & $\mathrm{As}$ \\
\hline 0.909 & 0.060 & 0.666 & 0.077 & 1.000 \\
1.000 & 0.000 & 0.500 & 0.641 & 0.161 \\
0.590 & 0.060 & 1.000 & 0.816 & 0.075 \\
0.272 & 0.020 & 0.916 & 0.000 & 0.064 \\
0.000 & 1.000 & 0.000 & 1.000 & 0.000 \\
\hline
\end{tabular}

表 5 江苏省五大湖泊水体中重金属综合评价结果

Tab.5 Evaluation results of heavy metal in water of the five lakes in Jiangsu Province

\begin{tabular}{ccccc}
\hline 湖泊 & $D_{i}^{+}$ & $D_{i}^{-}$ & $C_{i}$ & 排序 \\
\hline 太湖 & 1.3205 & 1.5484 & 0.5397 & 3 \\
滆湖 & 1.1412 & 1.3941 & 0.5499 & 4 \\
洪泽湖 & 1.3753 & 1.5055 & 0.5226 & 2 \\
高宝邵伯湖 & 0.9905 & 1.0272 & 0.5091 & 1 \\
骆马湖 & 1.0000 & 1.4142 & 0.5858 & 5 \\
\hline
\end{tabular}




\section{3 讨论}

在水资源的污染中, 重金属污染影响大, 可恢复难度大, 给江苏省的水环境带来很大的破坏. 重金属污 染物进人水中后通过一系列化学作用迁移转化, 参与和干扰各种环境化学过程和物质循环, 最终以一种或 多种形态长期存留在环境中, 造成永久性的潜在危害 ${ }^{[13]} .10$ 年内骆马湖水体 $\mathrm{Pb}$ 和 $\mathrm{Hg}$ 浓度最高, 洪泽湖水 体 Cd 浓度最高, 太湖水体 As 浓度最高. 对湖泊沉积物重金属的已有研究结果表明, 骆马湖受人为污染影响 严重的主要是重金属中的 $\mathrm{Pb}$ 与 $\mathrm{Cd}^{[14]}$, 洪泽湖底质污染为 $\mathrm{Cd}$ 污染 ${ }^{[15]}$, 危害太湖生态安全的主要重金属元 素为 $\mathrm{As} 、 \mathrm{Cr}$ 和 $\mathrm{Hg}^{[16]}$. 结合本研究结果证实, 沉积物与水体中重金属均可能与外源输人有关. 而且, 五大湖泊 水体中 $\mathrm{Cu}$ 和 $\mathrm{As}$ 浓度变化趋势大致相同(图 3). 已有研究资料表明, 太湖不同湖区/人湖河流水体重金属浓
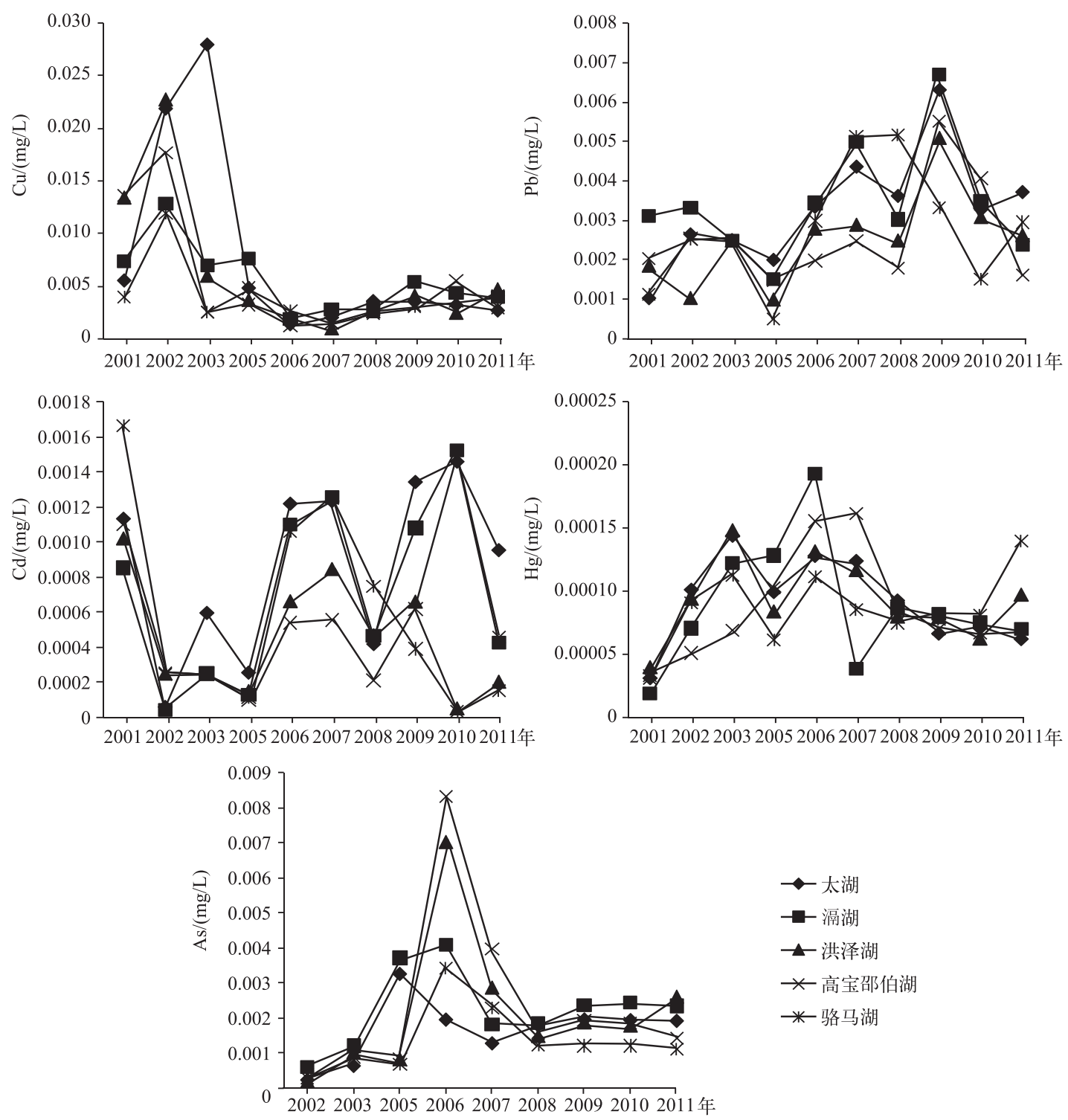

图 3 江苏省五大湖泊重金属浓度年度均值变化趋势

Fig. 3 Annual average concentrations of heavy metals in water of the five lakes in Jiangsu Province 
度存在一定的差异 ${ }^{[17]}$,但是本研究在方差分析的基础上,综合五大湖泊的信息,统计推断出 5 种重金属浓度 在湖泊内站位间均差异不显著. 然而, 江苏省五大湖泊重金属污染并未随着经济发展水平的差异呈现由北 到南越来越重的趋势, 这可能是由于各湖泊的利用方式不同, 湖泊周围环境条件不同, 以及湖泊监管治理的 不同,造成了江苏省五大湖泊水体中重金属浓度的差异. 但目前由于缺乏诸如湖泊周围的工业废物排放、农 用化肥使用等一些关联数据,因此有关湖泊之间重金属污染存在差异的原因有待于进一步探讨.

水体中的重金属污染主要来自两部分: 自然源和人为源. 自然源主要是岩石风化的碎屑产物,通过自然 途径进人水体中的重金属一般不会对水体造成污染; 人为污染源主要包括采矿和冶炼、金属加工、化工、废 电池处理、电子、造革和染料、农药和化肥的使用等, 是造成水体重金属污染的主要原因 ${ }^{[18]}$. 由于工农业生 产的迅猛发展, 排放到水体中的重金属元素日益增多, 水环境中的污染问题亦日益凸显. 因此, 控制重金属 污染物的排放, 改善江苏湖泊的水环境现状, 是摆在我们面前无法回避的一项十分迫切和艰巨的任务. 总体 而言, 10 年来江苏省五大湖泊水体重金属平均浓度均符合《渔业水质标准》,但本文发现湖泊间、站位内的 年份间重金属浓度存在一些差异, 有的差异已经达到统计学上显著的程度,这些结果可为检测管理部门以 及政府决策提供一些参考信息.

\section{4 结论}

经过 2001-2011 年对江苏省五大湖泊 1164 站次长期的定位监测, 表明五大湖泊水体中 $\mathrm{Cu} 、 \mathrm{~Pb} 、 \mathrm{Cd} 、 \mathrm{Hg}$ 和 As 浓度均符合《渔业水质标准》, 除 $\mathrm{Cu}$ 浓度外, 其他 4 种重金属浓度 5 个湖泊间均差异极显著, 湖泊内站 位间各重金属浓度均差异不显著. 综合分析结果表明, 高宝邵伯湖水体重金属污染程度最小, 骆马湖水体重 金属污染程度最大,洪泽湖、太湖和滆湖水体重金属污染综合评价优劣相当.

\section{5 参考文献}

[ 1 ] 誉培民, 王国祥, 李正鬼等. 健康水生态系统的退化及其修复一一理论、技术及应用. 湖泊科学, 2001, 13(3): 193-203. DOI 10.18307/2001.0301.

[2] 孟紫强. 环境毒理学基础. 北京: 高等教育出版社, 2005.

[ 3 ] 成 芳, 凌去非, 徐海军等. 太湖水质现状与主要污染物分析. 上海海洋大学学报, 2010, 19(1): 105-110.

[ 4 ] 李 波, 涭培民, 韩爱民. 洪泽湖水质的时空相关性分析. 湖泊科学, 2002, 14 (3)：259-266. DOI 10. 18307/2002. 0311.

[ 5 ] 高永年, 高俊峰, 陈坸烽等. 太湖流域水生态功能三级分区. 地理研究, 2012, 31(11): 1941-1951.

[6] 袁和忠, 沈 吉, 刘恩峰. 太湖重金属和营养盐污染特征分析. 环境科学, 2011, 32(3): 649-657.

[7] 刘瑞民, 王学军, 郑 一等. 太湖水质参数中小尺度空间结构特征. 长江流域资源与环境, 2002, 11(1): 32-35.

[8] 张少威, 刘茂松, 徐 驰等. 洪泽湖 7 条人湖河流的水质动态及其关联分析. 南京林业大学学报: 自然科学版, $2012,36(4)$ : 36-40.

[9] 李 波, 誉培民. 淮河流域及洪泽湖水质的演变趋势分析. 长江流域资源与环境, 2003, 12(1): 67-73.

[10］刘玉秀, 徐少青. TOPSIS 法用于医院工作质量的多指标综合评价. 中国卫生统计, 1993, 10(2): 12-15.

[11] 雷战波, 朱正威, 王 雷. 基于熵权夹角和 TOPSIS 的企业经济效益诊断模型. 运筹与管理, 2005, 14 (2): $142-148$.

[12] GB 11607-1989. 渔业水质标准.

[13] 戴树桂. 环境化学. 北京: 高等教育出版社, 1996.

[14] 王永平, 洪大林, 申 霞等. 骆马湖沉积物重金属及营养盐污染研究. 南水北调与水利科技, 2013, 11(6): 45-49.

[15］刘振坤, 张书海. 洪泽湖底质重金属污染变化趋势分析. 江苏环境科技, 2005, 18(4): 41-43.

[16] 石志芳. 太湖沉积物中重金属污染特征及生态风险评价 [学位论文]. 杨凌: 西北农林科技大学, 2010.

[17] Zeng J, Yang LY, Chen XF et al. Spatial distribution and seasonal variation of heavy metals in water and sediments of Taihu lake. Polish Journal of Environmental Studies, 2012, 21(5) : 1489-1496.

[18］王海东, 方凤满, 谢宏芳. 中国水体重金属污染研究现状与展望. 广东微量元素科学, 2010, 17(1): 14-18. 